\title{
Efficacy of Inhaled Salbutamol in the Management of Transient Tachypnoea of Newborn
}

\section{Shaukat Hussain, ${ }^{1}$ Muhammad Arslan Farooq, ${ }^{2}$ Muhammad Tayyab, ${ }^{3}$ Isfand Yar Khan ${ }^{4}$}

\begin{abstract}
Objective: To compare the outcome of salbutamol nebulization with controls (normal saline) in transient tachypnea of newborn.

Methods: In this randomized controlled trial 284 (142 in each group) neonates were included with gestational age above 35 weeks of any gender. Study was conducted in Department of Pediatrics Holy Family Hospital Rawalpindi. Sampling technique was consecutive non probability sampling. Duration of study was 6 months. Sample size had been calculated using WHO calculator; Significance level 5\%, Power of test 80\%, Population mean 5.4, Test value of population mean 4.8, Standard Deviation 1.8. At admission complete blood picture, C-reactive protein and chest $\mathrm{x}$-ray were done. Group-1 received salbutamol nebulization at a dose of $0.15 \mathrm{mg} / \mathrm{kg} /$ dose for 10 minutes in $2 \mathrm{ml}$ normal saline QID while Group-2 received only normal saline nebulization $2 \mathrm{ml}$ QID in 24 hours. Double blinding was ensured and in case of any side effects of salbutamol (tachycardia, arrhythmias and hypoglycemia) treatment was stopped immediately and managed accordingly.
\end{abstract}

Results: In this study, comparison of outcome of inhaled salbutamol with controls in transient tachypnea of newborn shows that respiratory rate per minute was 50.15+2.34 in Group-1 and 64.92+2.70 in Group-2, p value was 0.0001 , heart rate per minute in Group- 1 was recorded as $120.08+2.36$ and $134.79+3.27$ in Group2 , p value was 0.0001 , oxygen saturation(\%) was 97.27+0.92 in Group-1 and 83.28+1.81 in Group-2, p value was 0.0001 , duration of oxygen therapy(hours) was recorded as $15.49+1.84$ in Group-1 and 27.78+5.57 in Group-2, value of p was 0.0001 , duration of hospitalization(days) was recorded as $2.03+0.45$ in Group- 1 and $5.07+0.72$ in Group-2 while value of P was 0.0001

Conclusion: Salbutamol Nebulization is more effective than normal saline in TTN.

Key Words: Transient Tachypnea of newborn, management, inhaled salbutamol, outcome.

\section{Introduction:}

$\mathrm{M}$ ost common neonatal problem after birth is respiratory distress. ${ }^{1}$ One third of all neonates who needs admission after birth with respiratory distress are finally diagnosed as a case of transient tachypnea of newborn. Transient tachypnea of newborn results due to lung fluid collection, ultimately leading to pulmonary edema because of delay in the

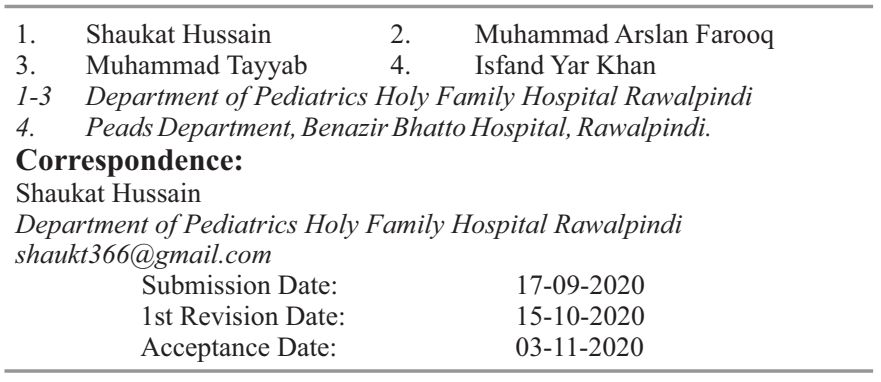

clearance of fluid. It usually settles down within 2-3 days after birth, but it may persist upto 5th day of life. ${ }^{1,2}$ It is very common in dyspneic neonates, with incidence of 4 to $5.7 \%$ among term infants, while it may occur upto $10 \%$ in pre mature neonates. ${ }^{3}$ Beta- 1 receptor stimulants like catecholamines and endogenous steroids play critical role in lung fluid absorption and level of these chemicals rise during process of delivery. Neonates with lower level of these endogenous chemicals suffer with decreased rate of clearance of lung fluid, which ultimately results in fluid collection at alveolar level. Most of the time TTN is self-remitting dieses but occasionally it may complicates resulting in severe hypoxia leading to death which is labeled as Malignant TTN. ${ }^{4,5}$ Management of TTN requires intensive supportive care unit. Aims of management is to maintain thermo-neutral environ- 
ment and provide adequate nutrition at a lower needs of oxygen supplementation. ${ }^{3-5}$ Few previous study suggest, management of TTN medications. This data suggested various medications including inhaled adrenaline, oral, inhaled or intravenous loop diuretics, Beta-2 receptor agonists and fluid restriction. But what is the most effective treatment for this disease is still controversial. ${ }^{4,5}$ Till date, no single effective drug option is available for this disorder except providing oxygen as a respiratory support. ${ }^{5}$ Neonates with this disorder who are intubated and put on ventilator can develop complications like hypotension and intra-ventricular bleeds. ${ }^{6-10}$

A study was conducted by M Mussavi et al on effects of bronchodilator in transient tachypnea of newborn. He concluded that $\beta 2$ agonist as compared to placebo causes a considerable improvement in the respiratory rate $(58.5 \pm 9.2$ vs $61.76 \pm 15.75)$, oxygen saturation $(97.6 \pm 1.9$ vs $98 \pm 1.6)$, oxygen therapy duration $(26 \pm 29.3$ vs $18.7 \pm 12.5)$ and treatment duration in days (5.4 \pm 2.4 vs $4.2 \pm 1.2){ }^{11}$

Mohammad Zadeh I et al observed the effect of salbutamol nebulization in TTN and result showed decreased duration of oxygen requirement, $p$-value 0.04 , hospitalization p-value 0.006 and onset of feeding with $\mathrm{p}$-value 0.03 , were significantly lower in salbutamol group when compared to placebo. ${ }^{12}$

Literature review regarding this has been lacking in our local setups. The rationale of this study is to see the efficacy of salbutamol nebulization as a treatment in TTN through RCT. The objective of this study is to assess changes in various parameters like duration of oxygen requirement, improvement of tachypnea and shortening of hospitalization in TTN, so that better outcome could be achieved with minimum morbidity.

\section{Methods}

In this randomized controlled trial 284 neonates were included with gestational age above 35 weeks of any gender. Study was conducted in Department of Pediatrics Holy Family Hospital Rawalpindi. Sampling technique is consecutive non probability sampling. Duration of study was 6 months. Sample size has been calculated using WHO calculator; Significance level 5\%, Power of test $80 \%$, Population mean 5.4, Test value of population mean 4.8, Standard Deviation $1.8, \mathrm{n}=142$ in each group. Informed consent was taken from the parents of all neonates. At the admission blood samples were collected for $\mathrm{CBC}$, CRP, Arterial Blood Gases. And Chest X-ray was done. Enteral feeding was withheld because of tachypnea and respiratory distress. Standard fluid was administered according to day of life and gestational age. Enrolled neonates were randomly placed into 2 groups; group 1 was treatment group and group 2 placebo group. Treatment group was receiving $0.15 \mathrm{mg} / \mathrm{kg} /$ dose Salbutamol in $2 \mathrm{~mL}$ of normal saline, and placebo group was receiving $2 \mathrm{~mL}$ of normal saline in QID for 24 hours. Each group was nebulized for 10 minutes by jet nebulizer. The oxygen inhalation was given according to the oxygen saturation of neonate, in order to maintain saturation above $90 \%$. Both drug and placebo solutions were prepared in similar shape and color coding by a health care person not involved in neonatal care in order to ensure double blinding. In case of any side effect (tachycardia or arrhythmias, hypoglycemia) treatment was stopped immediately and managed accordingly.

\section{Results}

Total 284 neonates (142 in each group) were included to compare the outcome of inhaled salbutamol with controls in transient tachypnea of newborn.

Sex distribution among participants shows that $51.41 \%(\mathrm{n}=73)$ in Group-1 and $50.70 \%(\mathrm{n}=72)$ in Group-2 were male and $48.59 \%(\mathrm{n}=69)$ in Group-1 and $49.30 \%(n=70)$ in Group-2 were females. (Table No. 1).

Gestational age of the patients was calculated as $18.31 \%(n=26)$ in Group- 1 and $19.72 \%(n=28)$ in Group-2 were between 35-37 weeks of gestation and $81.69 \%(n=116)$ in Group- 1 and $80.28 \%(n=114)$ in Group-2 had $>37$ weeks of gestation, mean + SD was calculated as $38.81+1.41$ weeks in Group-1 and $38.72+1.39$ weeks in Group-2. (Table No. 1)

Mean birth weight was $2350.493+111.90$ grams in Group-1 and 2347.88+88.09 grams in Group-2. (Table No. 1).

Comparison of outcome of inhaled salbutamol with controls in transient tachypnea of newborn shows that respiratory rate per minute was 50.15+2.34 in Group1 and $64.92+2.70$ in Group-2, p value was 0.0001 , heart rate per minute in Group-1 was recorded as 
$120.08+2.36$ and $134.79+3.27$ in Group-2, p value was 0.0001 , oxygen saturation(\%) was $97.27+0.92$ in Group-1 and $83.28+1.81$ in Group-2, p value was 0.0001 , duration of oxygen therapy(hours) was recorded as 15.49+1.84 in Group-1 and 27.78+5.57 in Group-2 value of P was 0.0001, duration of hospitalization(days) was recorded as $2.03+0.45$ in Group-1 and 5.07+0.72 in Group-2, value of $\mathrm{p}$ was 0.0001 . (Table No. 2).

Gestational age, sex distribution, birth weight, mode of delivery affecting final result were controlled by stratification for which independent sample t-test was applied.

\section{Discussion}

Transient tachypnea of the newborn (TTN), is a transient, self-remitting disease, that occurs due to decreased rate of lung fluid absorption in neonates. This delay in lung fluid clearance impairs the normal

Table 1: Distribution of Study Variables $(n=284)$

\begin{tabular}{ccc}
\hline Demographics Variables & $\begin{array}{c}\text { Group-1 } \\
(\mathbf{n = 1 4 2})\end{array}$ & $\begin{array}{c}\text { Group-2 } \\
(\mathbf{n = 1 4 2})\end{array}$ \\
\hline Gender: & $73(51.41 \%)$ & $72(50.70 \%)$ \\
Male & $69(48.59 \%)$ & $70(49.30 \%)$ \\
Female & & \\
Gestational Age (Weeks): & $26(18.31 \%)$ & $28(19.72 \%)$ \\
$35-37$ & $116(81.69 \%)$ & $114(80.28 \%)$ \\
$>37$ & $38.81 \pm 1.41$ & $38.72 \pm 1.39$ \\
Mean \pm SD & & \\
Birth weight (grams): & 2350.493 & 2347.88 \\
Mean & 111.90 & 88.09 \\
SD & & \\
Mode of delivery: & $50(35.12 \%)$ & $50(35.12 \%)$ \\
NVD & $92(64.78 \%)$ & $92(64.78 \%)$ \\
C-Section & &
\end{tabular}

Table 2: Comparison of Outcome of Inhaled Salbutamol with Controls in Transient Tachypnea of Newborn $(n=284)$

\begin{tabular}{|c|c|c|c|c|c|}
\hline \multirow[t]{2}{*}{ Outcome } & \multicolumn{2}{|c|}{$\begin{array}{c}\text { Group-1 } \\
(n=142)\end{array}$} & \multicolumn{2}{|c|}{$\begin{array}{c}\text { Group-2 } \\
(n=142)\end{array}$} & \multirow{2}{*}{$\begin{array}{c}\mathbf{P} \\
\text { Value }\end{array}$} \\
\hline & Mean & SD & Mean & SD & \\
\hline Respiratory rate/min & 50.15 & 2.34 & 64.92 & 2.70 & 0.0001 \\
\hline Heart rate/min & 120.08 & 2.36 & 134.79 & 3.27 & 0.0001 \\
\hline Oxygen saturation (\%) & 97.27 & 0.92 & 83.28 & 1.81 & 0.0001 \\
\hline $\begin{array}{l}\text { Duration of oxygen } \\
\text { therapy (hours) }\end{array}$ & 15.49 & 1.84 & 27.78 & 5.57 & 0.0001 \\
\hline $\begin{array}{l}\text { Duration of } \\
\text { hospitalization (days) }\end{array}$ & 2.03 & 0.45 & 5.07 & 0.72 & 0.0001 \\
\hline
\end{tabular}

physiology of transition of lungs from fetal to neonatal life. Beta-1receptor stimulants enhance this rate of lung fluid absorption.

Literature review regarding this has been lacking in our local setups. The rationale of this study was to see the efficacy of salbutamol nebulization as a treatment in TTN through RCT. The objective of this study is to assess changes in various parameters like duration of oxygen requirement, improvement of tachypnea and shortening of hospitalization in TTN, so that better outcome may be achieved with minimum morbidity.

We defined TTN on the basis of following clinical criteria: 1) $\geq 35$ weeks gestational age; 2) respiratory distress in less than 6 hours of life after birth (i.e., respiratory rate greater than $60 / \mathrm{min}$, grunting, nasal flaring, subcostal or intercostal retractions); 3) typical chest X-ray findings (i.e., fluid in transverse fissure, hyperinflation, prominent vascular/perihilar markings).

Our both study groups fulfilled the criteria of TTN. We excluded our study population from Meconium aspiration syndrome, Respiratory distress syndrome and Congenital pneumonia on the basis of clinical symptoms, signs, complete blood picture, c-reactive protein and chest $\mathrm{x}$-ray.

In our study, comparison of outcome of salbutamol nebulization with controls in transient tachypnea of newborn shows that respiratory rate per minute was $50.15+2.34$ in Group-1 and 64.92+2.70 in Group-2, p value was 0.0001 , heart rate per minute in Group-1 was recorded as $120.08+2.36$ and $134.79+3.27$ in Group-2, p value was 0.0001 , oxygen saturation(\%) was $97.27+0.92$ in Group-1 and $83.28+1.81$ in Group-2, p value was 0.0001 , duration of oxygen therapy (hours) was recorded as $15.49+1.84$ in Group-1 and 27.78+5.57 in Group-2, value of $p$ was 0.0001 , duration of hospitalization(days) was recorded as $2.03+0.45$ in Group- 1 and $5.07+0.72$ in Group-2, p value was 0.0001 .

Our data is supported by a study conducted by M Mussavi et al on effects of bronchodilator in transient tachypnea of newborn. He concluded that $\beta 2$ agonist as compared to placebo cause a considerable improvement in the respiratory rate $(58.5 \pm 9.2$ vs $61.76 \pm$ $15.75)$, oxygen saturation $(97.6 \pm 1.9$ vs $98 \pm 1.6)$, 